\title{
Story-Telling for Children in Image Processing Using Deep Learning - A Survey Review
}

\author{
P.Haritha ${ }^{\mathrm{a}, 1}$, S.Vimala ${ }^{\mathrm{b}}$ and S.Malathi ${ }^{\mathrm{b}}$ \\ ${ }^{a, b}$ Dept of CSE, Panimalar Engineering College, Chennai, India
}

\begin{abstract}
In this paper, a natural approach of the describing for the kids which it is brought into use, by making the image engraving technique of Deep Learning into thought. The previous work opens the technique for a course semantic mix plan (CSF) to mine the specialist features to encode the image content through thought instrument. A CSF benefits by three sorts of visual thought semantics which including the thing level, picture level, and spatial thought features, in three-stage course way. In the viewpoint on the current structure, the model takes the moved picture given by the customer as the information and starts recording the image what a PC find in human standard language with point by point explicit presents in the image appropriately exhibiting the significance of picture captioning. This strategy can be applied to the field of youngster's tutoring to make them pattern of adapting also entrancing. Fundamentally, we facilitate thought instrument with three kinds of features to orchestrate the setting data about the photos from differentviewpoints.
\end{abstract}

Keywords. instinctive methodology, CSF, three stage course, cycle of learning, deep learning, pc

\section{Introduction}

Deep learning is Associate in Nursing AI work that mirrors the operations of the human cerebrum in strategy information to be used in examination objects, perceiving discourse, interpreting dialects, and making choices. Profound learning AI is all through a grasp to travel looking out though not human bearing, drawing from information that is each unstructured and unlabelled. Profound Learning could likewise be an AI method upheld neural organization designs with numerous layers of strategy units, that has been effectively applied to an expansive arrangement of issues among the zones of picture acknowledgment and correspondence procedure. Facial acknowledgment includes abuse registering to distinguish human appearances in pictures or recordings, consequently menstruation explicit facial attributes. This may 
epitomize the hole among eyes, thus the general places of the nose, jaw line and mouth. This information is consolidated to make a facial mark, or profile. Pundits of veil acknowledgment conjointly expect that this new innovation might be inclined to assortment of the indistinguishable traps as face acknowledgment. A few of the training datasets utilized for biometric distinguishing proof are overwhelmed by Caucasian individuals. In the AI model, Convolutional Neural Network are created misuse Python, the CNN model is that the best fitted to picture data grouping. When the picture has been preprocessed by misuse A satisfactory apparatus, it will be served to the changed layers of the DCNN model. At spans the CNN model, there are quantities of covered up layers that is made ofneurons.

\section{Relatedwork}

Najatsmeda, Eva Dakich,"The effectiveness of digital storytelling in the classrooms: a comprehensive study",2017Advanced narrating is one of the creative academic methodologies that can draw in understudies in profound and significant learning. This examination project meant to establish a constructivist learning climate with advanced narrating. The exploration examined the academic parts of computerized narrating and the effect of advanced narrating on understudy realizing when instructors and understudies utilize computerized stories.

\subsection{JamshedMemon, Maira Sami, Rizwan Ahmed Khan,"Handwritten Optical Character Recognition(Ocr): A Comprehensive Systematic Literature Review (Slr)" 2020}

The goal of this survey paper is to sum up research that has been directed on character acknowledgment of manually written archives and to give research bearings. In this Systematic Li.They followed generally utilized electronic data sets by following precharacterized survey convention. Articles were looked through utilizing catchphrases, forward reference looking furthermore, in reverse reference looking to look through every one of the articles identified with the subject. This survey article serves the motivation behind introducing cutting edge results and methods on OCR and furthermore give research bearings by featuring researchholes.

\subsection{QIN WU, SIRUI WANG1, JIASHUO CAO1, "Object Recognition-Based Second Language Learning Educational Robot System for Chinese Preschool Children",2018}

In this paper, an instructive robot framework with object acknowledgment innovation is presented, which plans to give creative second language learning administrations for preschool youngsters in China. The proposed framework joins object acknowledgment and projection with English instructing and makes objects in everyday life more intriguing with extended liveliness to stand out for children. On the projection screen, kids can cooperate with a robot by contact or development and can undoubtedly trigger more intelligent impacts. To assess the viability of the proposed framework, we directed a trial, and the outcomes showed that the framework can improve the language. 
2.3. Vanessa Echeverria, "Towards data storytelling to support teaching and learning", 2017

They present a pilot study that investigates the viability of these DS components dependent on instructors' reactions to paper models. The double design is understanding the commitment of each visual component for information narrating, and the viability of the upgrades when joined. The outcomes recommend that DS components could add clearness, particularly when there are different potential stories in a mind bogglingrepresentation.

2.4. Michael Paul C. Gutierrez, "Digital Storytelling vs Traditional Storytelling: Teaching English Language to ANHS Students", 2019

This paper will zero in on figuring out which kind of narrating will be more successful in showing English: customary or computerized. Customary narrating is the narrator audience approach, while advanced narrating is narrating utilizing PC based apparatuses. These two sorts of narrating were utilized to test the English capability of 2 distinct areas from the Adult Night High School (ANHS) understudies of La Salle Green Hills. Utilizing a pre-test acquired from the Word-Up project, the analysts had the option to evaluate their underlying English capability level. Subsequently, the understudies were approached to peruse the story 'The Monkey's Paw' by W.W Jacobs, one through customary perusing and the other through PowerPoint introduction.

\section{Architecture}

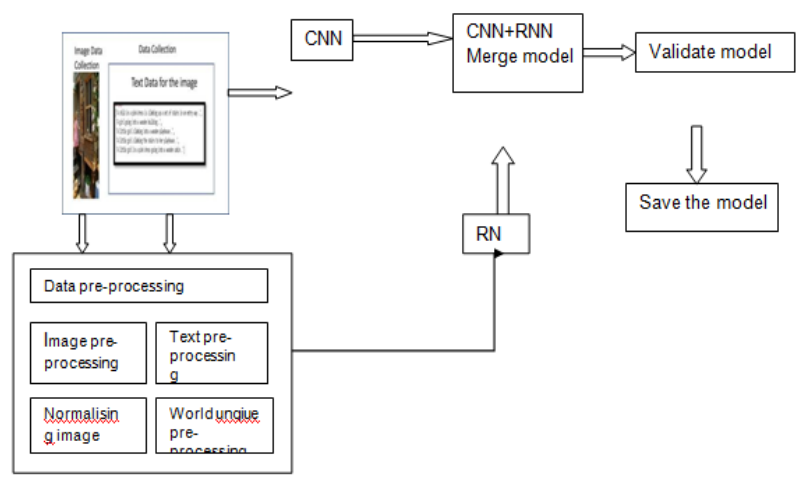

Figure 1. Story telling For kids architecture

In the current framework, the substance of the picture is encoded utilizing a few consideration methodology to remove the highlights. The structure is assembled utilizing Cascade Semantic Fusion engineering in a three phases: In the principal stage, object-level consideration highlights are removed dependent on the pre-prepared locator. In second stage consolidates object level consideration highlights with spatial highlights, along these lines instigating picture level consideration highlights to enhance the setting data around the items. In conclusive stage finds the striking locale portrayal making the thought moreproficient. 


\section{Result AndDiscussion}

Data Collection is the initial step in the process of evaluating the outcomes and the collected dataset is pre-processed to remove any noise or outliers if present. After completing data cleaning process the data will be Rescale and Discretize for next process.
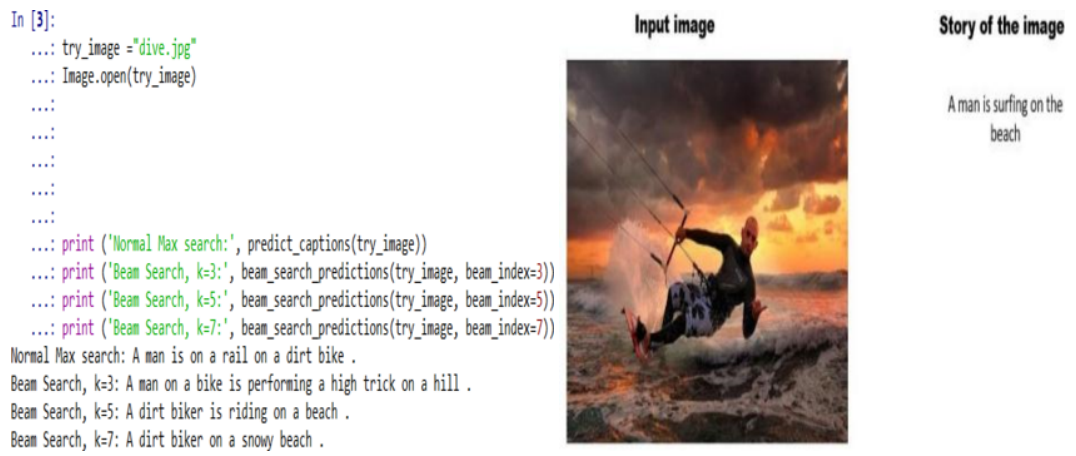

Figure 2 .Output screenshot for the input image1

\section{PerformanceAnalysis}

The significance execution grid is a way to deal with focusing on which parts of execution ought to be dependent upon progress. In this system we get $92 \%$ of accuracy by training the dataset. Let us see the performance difference between existing system and proposed system in table 1:

Table 1.Performance analysis

\begin{tabular}{llll}
\hline Existing method & $\begin{array}{l}\text { Data } \\
\text { flicker(8k) }\end{array}$ & setEpoch 25 & Accuracy 75 \\
Proposed method & $\begin{array}{l}\text { Data } \\
\text { flicker(8k) }\end{array}$ & setEpoch 25 & Accuracy 92 \\
\hline
\end{tabular}

\section{Conclusion}

Datasets are a vital piece of the field of AI. The Major advances in this field can result from progresses in learning calculations, PC equipment, and, less-instinctively, the accessibility of top notch preparing datasets. When the model is stacked with the pictures given by the client, at that point the frame work begins in scribing accordingly shaping an account. An intriguing story can be acquired with the client inputted pictures. By putting hours of training for the proposed system $92 \%$ accuracy have been achieved. In each epoch, accuracy increase rate was very minimum. We have trained for nearly 8000 images with respective to the different image captioning. The proposed model for $92 \%$ its prediction for the image was fair. 


\section{Future goal}

Narrating is an extraordinary social and social movement of offering stories to an expect to teach, engage, and impart virtues which are for the most part utilized in the social oral narrating occasions. Computerized narrating engages understudies to be sure communicators and makers of media as they acquire fundamental 21st-century proficiency abilities and arrive at more deep comprehension altogether zones of the educational plan. In future it will be used in education.

\section{References}

[1] Najatsmeda, Eva Dakich, The effectiveness of digital storytelling in the classrooms: a comprehensive study, 2017.

[2] Jamshed Memon,Maira Sami, Rizwan Ahmed Khan.Handwritten Optical Character Recognition(OCR): A Comprehensive Systematic Literature Review (SLR) ,2020.

[3] Qin Wu, Sirui Wang, Jiashuo Cao. Object Recognition-Based Second Language Learning Educational Robot System for Chinese Preschool Children .2018.

[4] Vanessa Echeverria.Towards data storytelling to support teaching and learning .2017.

[5] Akshaya.B, M.Rajendiran .Securing Documents Using Digital Certificate-A Review.International Journal of Research in Advent Technology (IJRAT). 2018.

[6] S.Hema Kumar, J.UdayKiran, V.D.AKumar, G.Saranya, Ramalakshmi V .Effective Online Medical Appointment System. International Journal of Scientific \& Technology Research. Volume 8. Issue 09.September 2019. Pages $803-805$.

[7] Ambeth Kumar.V.D, Dr.M.Ramakrishnan, V.D.Ashok Kumar and Dr.S.Malathi (2015) "Performance Improvement using an Automation System for Recognition of Multiple Parametric Features based on Human Footprint. kuwait journal of science. Vol 42, No 1 (2015). pp:109-132.

[8] Ambeth Kumar.V.D, V.D.Ashok Kumar, S.Malathi, K.Vengatesan, M.Ramakrishnan. Facial Recognition System For Suspect Identification Using A Surveillance Camera .Pattern Recognition and Image Analysis (Springer), Volume 28, Issue 3, pp 410-420, $2018 . \quad$ (DOI: 10.1134/S1054661818030136)

[9] Ambeth Kumar.V.D and M.Ramakrishan .Employment Of Footprint Recognition System. in the month of December for Indian Journal of Computer Science and Engineering (IJCSE) Vol. 3 No.6 Dec 2013

[10] Ramya.T,Dr.Malathi,S.,ratheeksha,G.R. and Dr.V.D.Ambeth Kumar (2014).Personalized authentication procedure for restricted web service access in mobile phones. Applications of Digital Information and Web Technologies (ICADIWT), 2014, Page(s):69 - 74, Bangalore, India (ISBN:978-14799-2258-1)

[11] Ambeth Kumar.V.D (2018).Novel Wireless Sensing System For The Welfare Of Sewer Laborers", Healthcare Technology Letters (IET) Volume 5, Issue 4, p. $107-112$.

[12] Ambeth Kumar.V.D, G.Saranya , D.Elangovan, V.RahulChiranjeevi, V.D.Ashok Kumar.IOT Based Smart Museum Using Wearable Device .Lecture Notes in Networks and Systems, Vol.55, pp: 33-42, 2018

[13] Ambeth Kumar.V.D and Dr.M.Ramakrishan(2011).Footprint Based Recognition System.in the month of April for the International Journal Communication in Computer and Information System (CCIS) Journal (Springer) Volume 147, Part 3, 358-367, DOI: 10.1007/978-3-642-20573-6_63, 2011

[14] Ambeth Kumar.V.D (2017).Efficient Routing for Low Rate Wireless Network a Novel Approach. International Journal of Image Mining, Vol. 2, Nos. 3/4, 2017, 2017

[15] Ambeth Kumar.V.D, V.D.Ashok Kumar, Dr.S.Malathi and P.Jagaeedesh, (2014) .Intruder Identification using Footprint Recognition with PCA and SVM Classifiers for the International Journal of Advanced Materials Research Vols.1345, PP 984-985 (2014) pp 1345-1349. [DOI:10.4028/www.scientific.net/AMR.984-985.1345]

[16] Ambeth Kumar.V.D, G.Gokul, S.Malathi, K.Vengatesan, D.Elangovan, B.Chitra .Implementation Of The Pulse Rhythemic Rate For The Efficient Diagonising Of The Heart Beat . Healthcare Technology Letters (IET) 2019 Apr 17;6(2):48-52.

[17] S.V. Ruphitha et.al, . Management of Major Postpartum Haemorrhage by using Zigbee protocol - A Review . 2021 6th International Conference on Inventive Computation Technologies (ICICT) (DOI: 10.1109/ICICT50816.2021.9358757) 